\title{
Nuclear Dynamics in the Vicinity of a Crossing Seam: Vibrational Spectrum of $\mathrm{HD}_{2}$ Revisited
}

\author{
Z. R. XU, A. J. C. VARANDAS \\ Departamento de Quimica, Universidade de Coimbra, P-3049 Coimbra Codex, Portugal
}

Received 19 May 2000; revised 5 February 2001; accepted 5 March 2001

\begin{abstract}
So far, most studies of the geometric phase effect have presumed that the phase is path-independent; hence, one must supply another restriction concerning the boundary condition on the nuclear wave functions when dealing with nonsymmetric isotopomers of $\mathrm{X}_{3}$ systems. We report calculations of the vibrational spectra of $\mathrm{HD}_{2}$ using a recently proposed generalized Born-Oppenheimer (GBO) formalism. The calculations demonstrate that there are significant differences between the results calculated from the present GBO method and those based on the preceding presumption. (c) 2001 John Wiley \& Sons, Inc. Int J Quantum Chem 83: 279-285, 2001
\end{abstract}

Key words: generalized Born-Oppenheimer; vibrational spectra; $\mathrm{HD}_{2}$; nonsymmetric isotopomers

\section{Introduction}

n 1963, Herzberg and Longuet-Higgins [1] proved that a real-valued adiabatic electronic wave function changes sign when the nuclear coordinates traverse a closed path that encircles a conical intersection. This change implies a breakdown of the standard Born-Oppenheimer (BO) treatment [2] whenever such an intersection is present [1,3-8]. Two decades ago, Mead and Truhlar [9-11] showed that the single-surface $\mathrm{BO}$ treatment could be generalized by introducing a vector potential into the nuclear Schrödinger equation. A few years later,

Correspondence to: A. J. C. Varandas.

Contract grant sponsor: Fundação para a Ciência e Tecnologia.

International Journal of Quantum Chemistry, Vol. 83, 279-285 (2001)

(C) 2001 John Wiley \& Sons, Inc.
Berry [12] proved the geometric phase (GP) effect (which had then become known also as the Berry phase effect) in a wider context by showing that it can be present on the adiabatic evolution of other quantum systems. Moreover, Aharonov and Anandan [13] removed the restriction of adiabaticity and defined the geometric phase for any cyclic quantum evolution. They also explained the AharonovBohm [14] effect for a charged particle moving in the presence of a magnetic solenoid, which is often $[15,16]$ taken to be synonymous with the GP effect due to the similarity of the involved differential equations.

It is well established that the GP effect plays a significant role in molecular spectra [17-21] and scattering [22-29]; for reviews, see Refs. [15] and [16] (see also Ref. [30] and references therein). In fact, 
as shown many years ago [31] by ab initio calculations, the GP effect may be present even when the system has no symmetry, such as is the case for the ground electronic state of LiNaK. Two alternative possibilities have been suggested to account for the GP effect in the case of an $X_{3}$ molecule. One consists of multiplying the real double-valued electronic wave functions by a complex phase factor that changes sign when it encircles the conical intersection and, hence, makes the resulting complex electronic wave function single-valued [24, 32, 33]. In fact, such a complex phase factor leads to the previously mentioned vector potential of Mead and Truhlar $[9,11]$. The other approach is owing to Billing and Marković [34], who utilized hyperspherical coordinates to include the GP effect in $\mathrm{X}_{3}$ molecules that have a single $D_{3 h}$ conical intersection seam. A similar method was employed by us $[17,20,21]$ to study the resonance and vibrational spectra of $\mathrm{H}_{3}$ and $\mathrm{Li}_{3}$; this work was recently reviewed [35]. The most recent $J=0$ calculations of the vibrational states of $\mathrm{Li}_{3}$ in its lowest electronic doublet state, performed using a reliable double many-body expansion $[36,37]$ potential energy surface $[38,39]$ and a minimum-residual filter diagonalization (MFD) technique [40], both without consideration (NGP) and with consideration (GP) of the GP effect, can be found elsewhere [20, 21]. Of course, $\mathrm{H}_{3}$ and its isotopic variants also have been very extensively studied $[17,20,21,25,26,41-50]$. In particular, the articles by Varandas and Yu $[17,47]$ focused on the role of the GP effect in the transition state resonances and vibrational states of $\mathrm{H}_{3}$, which were investigated using a time-dependent wavepacket approach. However, a complication arises when the GP effect is investigated in isotopomers of such $\mathrm{X}_{3}$ systems. This complication is due to the mass scaling involved in defining the hyperspherical coordinates. Kuppermann and $\mathrm{Wu}$ [25] studied the GP effect in $\mathrm{DH}_{2}$ using a mass-scaled Jacobi vectors [51] formula. More recently, we proposed a novel split basis technique [52] to treat the same problem. In these treatments, the geometric phase is assumed to be path-independent and, hence, another restriction must be added to the boundary condition on the nuclear wave functions for nonsymmetric isotopomers of $X_{3}$ systems.

Recently, we followed a strategy similar to Baer and Englman [53, 54], and derived novel singlesurface Born-Oppenheimer equations [49]. (A rebuttal to their work was published [55], but the arguments involved do not concern our work. Thus, we admit our formalism to be strictly valid in the vicinity of the conical intersection, but convey generality to it by invoking the well known [1, 4-7] fact that such regions influence the nuclear dynamics in a dominant way, even when energetics allows us to sample areas of configurational space far away from the crossing seam.) We found [49] that the geometric phase $A(\mathbf{R})$ is defined by the argument of the complex electronic vector state in the complex plane spanned by the two real-valued electronic components. Such an angle is identical (up to a constant) to the mixing angle $\gamma(\mathbf{R})$ in the two coupled-state problem. By employing a line-integral technique $[54,56-66]$ to study the GP effect in two coupled-state hydrogenic systems, we also showed [50] that the adiabatic-diabatictransformation angle $[56-59,61]$ is identical (up to a constant) to the geometry-dependent mixing angle $\gamma(\mathbf{R})$ of the orthogonal transformation that diagonalizes the diabatic potential matrix [47, 67-71]. Most recently, we discussed [72] the singularities that arise in the Hamiltonian at the crossing seam and we established the relationship between the magnetic vector, the electric scalar gauge potentials, and the mixing angle.

This article is organized as follows. In the next section, we briefly survey the theory. Using the relevant generalized Born-Oppenheimer (GBO) equation, calculations of the vibrational spectrum for $\mathrm{HD}_{2}$ are reported in the third section. The conclusions are given in last section.

\section{Theory \\ GENERALIZED BORN-OPPENHEIMER EQUATION}

The time-independent Schrödinger equation (relativistic effects are ignored) for a coupled multistate electronic manifold assumes the form $[9,48,53,54$, $73,74]$

$\left\{-\frac{\hbar^{2}}{2 \mu}\left[\nabla^{2}+2 \mathbf{F}(\mathbf{R}) \cdot \nabla+\mathbf{G}(\mathbf{R})\right]+\mathbf{V}(\mathbf{R})\right\} \chi(\mathbf{R})=\mathbf{E} \chi(\mathbf{R})$,

where $\mu=\left[(1 / M) \prod_{i=1}^{N} m_{i}\right]^{1 /(N-1)}$ is the characteristic reduced mass of the system, $\chi(\mathbf{R})$ denotes a column vector whose components are the nuclear wave functions $\chi_{I}(\mathbf{R})$, and the matrix elements of $\mathbf{F}(\mathbf{R}), \mathbf{G}(\mathbf{R})$, and $\mathbf{V}(\mathbf{R})$ are defined by

$$
\begin{aligned}
& \mathbf{F}_{I J}(\mathbf{R})=\left\langle\psi_{I}(\mathbf{r} ; \mathbf{R}) \mid \nabla \psi_{J}(\mathbf{r} ; \mathbf{R})\right\rangle, \\
& G_{I J}(\mathbf{R})=\left\langle\psi_{I}(\mathbf{r} ; \mathbf{R}) \mid \nabla^{2} \psi_{J}(\mathbf{r} ; \mathbf{R})\right\rangle, \\
& V_{I J}(\mathbf{R})=\left\langle\psi_{I}(\mathbf{r} ; \mathbf{R})\left|H_{e}\right| \psi_{J}(\mathbf{r} ; \mathbf{R})\right\rangle,
\end{aligned}
$$


where (and hereafter) the bra-ket notation $\langle\mid\rangle$ is used to specify integration over the electronic coordinates $\mathbf{r}$ only, and $\nabla$ implies taking the gradient with respect to the nuclear internal degrees of freedom R. After some algebraic manipulation, Eq. (1) may assume the simplified single-surface equation form

$$
\begin{aligned}
\left\{-\frac{\hbar^{2}}{2 \mu}\left[\nabla^{2}-\right.\right. & \left.\left.(\nabla \gamma(\mathbf{R}))^{2}\right]+V_{2}-E\right\} \tilde{\chi} \\
= & -i \frac{\hbar^{2}}{2 \mu}\left[\nabla^{2} \gamma(\mathbf{R})+2 \nabla \gamma(\mathbf{R}) \cdot \nabla\right] \tilde{\chi},
\end{aligned}
$$

where, following Longuet-Higgins [5] and Baer and Englman [53], we have defined the complex nuclear wave function $\tilde{\chi}$ as

$$
\tilde{\chi}=\frac{1}{\sqrt{2}}\left(\chi_{1}+i \chi_{2}\right) .
$$

Note that to obtain Eq. (5), we have neglected the term $\left(V_{1}-V_{2}\right) \chi_{1} / \sqrt{2}$ based on the assumption that the dynamics is dominated by the behavior of the two potential energy surfaces in the vicinity of the conical intersection (where $V_{1} \simeq V_{2}$ ). Note also that $V_{2}$ stands for the upper adiabatic potential energy surface and that Eq. (5) leads to the BornOppenheimer approximation when the derivative coupling elements are constant or zero. We further point out that Eq. (5) differs from an equation derived earlier by Baer and co-workers $[53,75]$ for the lower adiabatic sheet in that the mixing angle now appears in it explicitly. Moreover, because Eq. (5) has been derived under the assumption that the dynamics is controlled by the vicinity of the conical intersection, it should be valid to describe also the nuclear dynamics in the upper adiabatic potential energy surface provided that the appropriate adiabatic potential function is used.

Similarly to the case of the nuclear wave function, Eq. (6), we now define the complex electronic wave function (for the use of a similar definition, see Ref. [76]) as

$$
\tilde{\psi}=\frac{1}{\sqrt{2}}\left(\psi_{1}+i \psi_{2}\right),
$$

where $\psi_{I}$ are the real-valued electronic wave functions that correspond to the wave functions $\chi_{I}$ for the Ith nuclear state. In the complex plane spanned by the two electronic vector states, $\left\{\left|\psi_{1}\right\rangle, i\left|\psi_{2}\right\rangle\right\}$, the complex vector state $|\tilde{\psi}\rangle$ has, therefore, an argument equal to $A(\mathbf{R})=\arg |\tilde{\psi}\rangle$. Of course, the same is true for the complex nuclear wave functions: in the complex plane $\left\{\left|\chi_{1}\right\rangle, i\left|\chi_{2}\right\rangle\right\}$, the complex vector state $|\tilde{\chi}\rangle$ is characterized by the same argument $A(\mathbf{R})=$ $\arg |\tilde{\chi}\rangle$ as $|\tilde{\psi}\rangle$.

We proceed by evaluating the derivative coupling for the complex electronic wave function defined in Eq. (7). We get [49]

$$
\left\langle\psi_{1} \mid \nabla \psi_{2}\right\rangle=\nabla A(\mathbf{R}),
$$

which shows that the derivative coupling is given by the gradient of the geometric phase. A similar result (except for the sign) was obtained by Baer [54] using a different approach. Of course, Eq. (8) holds exactly in the vicinity of the crossing seam where the phase $A(\mathbf{R})$ obeys the relationship $A(\mathbf{R})=A_{1}(\mathbf{R})=$ $A_{2}(\mathbf{R})$. On the other hand, it is easy to show [49] that

$$
\left\langle\psi_{1} \mid \nabla \psi_{2}\right\rangle=\nabla \gamma(\mathbf{R}) .
$$

By comparing Eq. (9) with Eq. (8), we then get

$$
\nabla A(\mathbf{R})=\nabla \gamma(\mathbf{R}) .
$$

This is a key result for the present work, because it shows that the geometric phase $A(\mathbf{R})$ is identical to the mixing angle $\gamma(\mathbf{R})$ except for a constant term that has no physical implications. Thus, provided that we chose such a constant term to be zero, we get

$$
A(\mathbf{R})=\gamma(\mathbf{R}) .
$$

We now recall [50] that the mixing angle $\gamma(\mathbf{R})$ has the correct sign-change behavior: $\Delta \gamma=\pi$ for a closed path that encircles the crossing seam; $\Delta \gamma=0$ for a closed path that does not encircle such a seam. Note that the phase $A(\mathbf{R})$ corresponds to the phase change accumulated over a cyclic path where the dynamical phase vanishes [13, 77, 78], and this will be denoted the geometric phase. Note further that the phase defined from Eq. (11) will display the correct sign-change behavior. In other words, $\gamma(\mathbf{R})$ can be used to avoid artificial impositions of proper boundary conditions in the nuclear wave functions, as most authors have done so far.

\section{Vibrational Spectrum of $\mathrm{HD}_{2}$}

\section{CROSSING SEAM OF POTENTIAL ENERGY SURFACES}

As shown elsewhere [50], for any isotopomer of a $\mathrm{X}_{3}$ system, the crossing seam in hyperspherical coordinates $(\rho, \theta, \phi)$ is generally defined at an arbitrary value of the hyperradius $\rho$ by

$$
\begin{aligned}
\phi_{s}=\tan ^{-1}( & \cos \chi_{A C}-t \cos \chi_{A B}-\left(d_{A} / d_{C}\right)^{2} \\
& \left.+t\left(d_{A} / d_{B}\right)^{2}\right)\left(\sin \chi_{A C}+t \sin \chi_{A B}\right)^{-1}
\end{aligned}
$$




$$
\theta_{s}=2 \sin ^{-1}\left\{\frac{\left(d_{A} / d_{B}\right)^{2}-1}{\cos \left(\phi_{s}-\chi_{A B}\right)-\left(d_{A} / d_{B}\right)^{2} \cos \phi_{s}}\right\},
$$

where $t$ is given in the form

$$
t=\left[\left(\frac{d_{A}}{d_{C}}\right)^{2}-1\right]\left[\left(\frac{d_{A}}{d_{B}}\right)^{2}-1\right]^{-1}
$$

and

$$
\begin{array}{ll}
d_{X}^{2}=\frac{m_{X}}{\mu}\left(1-\frac{m_{X}}{M}\right), & \chi_{X Y}=2 \tan ^{-1}\left(\frac{m_{Z}}{\mu}\right), \\
\mu=\sqrt{\frac{m_{A} m_{B} m_{C}}{M}}, & M=m_{A}+m_{B}+m_{C}
\end{array}
$$

with $X, Y$, and $Z$ standing for atoms $A, B$, and $C$ with masses $m_{A}, m_{B}$, and $m_{C}$. In case two atomic masses are equal, namely $m_{B}=m_{C}$, we get for $\theta_{s}$ the simplified expression

$$
\theta_{s}=2 \sin ^{-1}\left|\frac{m_{B}-m_{A}}{m_{B}+2 m_{A}}\right|,
$$

whereas $\phi_{s}$ assumes the value $\pi$ when $m_{A}>m_{B}$ and assumes the value zero when $m_{A}<m_{B}$. For the case of $\mathrm{HD}_{2}$, the equation for the (straight line) seam is therefore defined [50] by $\theta_{s}=0.5048 \mathrm{rad}$, $\left.\phi_{s}=0\right)$. Since $\theta_{s}$ is different from zero, only closed paths with $\theta \geq \theta_{s}$ will enclose the seam: all other loops that correspond to $\theta<\theta_{s}$ will not satisfy such a requirement.

\section{CALCULATIONS AND DISCUSSION}

The rovibrational energy states can be calculated by solving Eq. (5), namely

$$
\hat{H} \tilde{\chi}=E \tilde{\chi},
$$

where $\hat{H}=\hat{H}_{0}+i \hat{H}_{1}$, where $\hat{H}_{0}$ is the system Hamiltonian without consideration of the GP effect. In modified hyperspherical coordinates [17, 79] (see also Refs. [80] and [81]) and neglecting all spin-orbit and spin-spin interactions, we have

$$
\begin{aligned}
\hat{H}_{0}= & -\frac{\hbar^{2}}{2 \mu}\left\{\frac{\partial^{2}}{\partial \rho^{2}}+\frac{16}{\rho^{2}}\left[\frac{1}{\sin \theta} \frac{\partial}{\partial \theta} \sin \theta \frac{\partial}{\partial \theta}\right.\right. \\
& \left.\left.+\frac{1}{4 \sin ^{2}(\theta / 2)} \frac{\partial^{2}}{\partial \phi^{2}}\right]\right\} \\
& +\frac{\hat{J}^{2}-\hat{J}_{z}^{2}}{\mu \rho^{2} \cos ^{2}(\theta / 2)}+\frac{\hat{J}_{z}^{2}+4 i \hbar \hat{J}_{z} \cos (\theta / 2)(\partial / \partial \phi)}{2 \mu \rho^{2} \sin ^{2}(\theta / 2)} \\
& +\frac{15 \hbar^{2}}{8 \mu \rho^{2}}+\frac{\sin (\theta / 2)}{\mu \rho^{2} \cos ^{2}(\theta / 2)} \frac{1}{2}\left[\hat{J}_{+}^{2}+\hat{J}_{-}^{2}\right] \\
& +V_{2}(\rho, \theta, \phi)+\frac{\hbar^{2}}{2 \mu}[\nabla \gamma(\rho, \theta, \phi)]^{2},
\end{aligned}
$$

where $\hat{J}, \hat{J}_{z}$, and $\hat{J}_{ \pm}$are the total angular momentum, its component along the $z$ axis, and the raising/lowering operators in the body-fixed frame, respectively, which are defined by the set of external coordinates $(\alpha, \beta, \gamma)$. In turn, $V_{2}$ is the potential energy surface of the system, which depends only on the three internal coordinates $(\rho, \theta, \phi)$. The hyperangles $\theta$ and $\phi$ are related [17] to the Smith-Whitten $(\Theta, \Phi)$ hyperangles [82] through the relationships $\theta=\pi-4 \Theta$ and $\phi=2 \pi-2 \Phi$. Hence, the range of $\theta$ is extended from 0 to $\pi$ and, hence, Legendre (or Jacobi) polynomials in $\cos \theta, \varphi_{j}(\cos \theta)$, can be used as the finite basis representation in $\theta$. To include the GP effect, we employ the generalized Born-Oppenheimer formulation described earlier, i.e., we take into consideration the imaginary operator $i \hat{H}_{1}$, where

$$
\hat{H}_{1}=-\frac{\hbar^{2}}{2 \mu}\left[\nabla^{2} \gamma(\rho, \theta, \phi)+2 \nabla \gamma(\rho, \theta, \phi) \cdot \nabla\right] .
$$

Note that we can still use Eq. (17) in the present case because the dynamics should be dominated by the GP effect which is dictated by the conical intersection. Indeed, even if this is not the case, we note that all previous GP calculations have been based on a similar type judgement.

Following previous work $[20,21]$, the action of the Hamiltonian $\hat{H}$ on the wave function (Lanczos vector) was carried out using a mixed grid-basis method. Uniform grids were employed for the coordinates $\rho$ and $\phi$, while the actions of the associated kinetic energy operators were performed by using a prime-factor fast Fourier transform technique [17]. Calculations of the eigenenergies were carried out by using the MFD technique. Similarly to the simple Lanczos algorithm, the MFD approach has low storage requirements (only two iteration vectors) and utilizes just a single Lanczos recursion for the eigenvalue problem. Furthermore, it can eliminate spurious and ghost eigenvalues [83].

In addition to the NGP calculations reported before [52], we present two sets of novel results: one uses the present formalism (set I) and the other is based on the common assumption that $A(\mathbf{R})=\phi / 2$ (set II) by using a split basis approach [52]. In this case, for $\theta<\theta_{s}$, we expand the nuclear wave function in terms of a single-valued basis

$$
\chi(\rho, \theta, \phi)=\sum_{n} C_{n}(\rho) f(\theta) \cos (n \phi),
$$

where $C_{n}$ are expansion coefficents and $f(\theta)$ is a $\theta$-dependent orthonormal polynomial type basis. Instead, for $\theta>\theta_{s}$, we carry out the expansion using 
the doubled-valued basis

$$
\chi(\rho, \theta, \phi)=\sum_{m} C_{m}(\rho) f(\theta) \sin \left[\left(m+\frac{1}{2}\right) \phi\right],
$$

where $C_{m}$ are also expansion coefficients. The calculated numerical values are reported in Tables I and II for the lowest 100 calculated levels.

The differences between these two sets of GP calculations are illustrated graphically in Figure 1 and also compared with the NGP calculations. As can be seen, there are differences between them: In set I, using Eq. (17), we have 50 levels below the energy value of $4.7850 \mathrm{eV}$, whereas in set II, only 49 levels appear. Note that level number 17, which corresponds to an energy of $4.5054 \mathrm{eV}$, is missing in set II.

TABLE I

Vibrational energy levels (in electronvolts) of $\mathrm{HD}_{2}$ calculated using Eq. (17).

\begin{tabular}{rrrrrr}
\hline 1 & 3.5939 & 35 & 4.7650 & 69 & 4.8107 \\
2 & 3.7591 & 36 & 4.7661 & 70 & 4.8124 \\
3 & 3.9150 & 37 & 4.7686 & 71 & 4.8134 \\
4 & 4.0614 & 38 & 4.7697 & 72 & 4.8154 \\
5 & 4.0851 & 39 & 4.7727 & 73 & 4.8169 \\
6 & 4.1158 & 40 & 4.7729 & 74 & 4.8186 \\
7 & 4.1981 & 41 & 4.7750 & 75 & 4.8215 \\
8 & 4.2285 & 42 & 4.7758 & 76 & 4.8231 \\
9 & 4.2571 & 43 & 4.7760 & 77 & 4.8251 \\
10 & 4.3248 & 44 & 4.7776 & 78 & 4.8262 \\
11 & 4.3606 & 45 & 4.7803 & 79 & 4.8284 \\
12 & 4.3870 & 46 & 4.7807 & 80 & 4.8304 \\
13 & 4.4407 & 47 & 4.7812 & 81 & 4.8315 \\
14 & 4.4475 & 48 & 4.7830 & 82 & 4.8338 \\
15 & 4.4810 & 49 & 4.7837 & 83 & 4.8351 \\
16 & 4.4974 & 50 & 4.7843 & 84 & 4.8368 \\
17 & 4.5054 & 51 & 4.7854 & 85 & 4.8392 \\
18 & 4.5449 & 52 & 4.7864 & 86 & 4.8408 \\
19 & 4.5564 & 53 & 4.7873 & 87 & 4.8426 \\
20 & 4.5718 & 54 & 4.7885 & 88 & 4.8449 \\
21 & 4.5897 & 55 & 4.7901 & 89 & 4.8462 \\
22 & 4.6114 & 56 & 4.7912 & 90 & 4.8475 \\
23 & 4.6157 & 57 & 4.7925 & 91 & 4.8491 \\
24 & 4.6360 & 58 & 4.7930 & 92 & 4.8513 \\
25 & 4.6656 & 59 & 4.7945 & 93 & 4.8539 \\
26 & 4.6815 & 60 & 4.7967 & 94 & 4.8553 \\
27 & 4.6852 & 61 & 4.7977 & 95 & 4.8568 \\
28 & 4.7037 & 62 & 4.7999 & 96 & 4.8590 \\
29 & 4.7115 & 63 & 4.8010 & 97 & 4.8616 \\
30 & 4.7193 & 64 & 4.8026 & 98 & 4.8622 \\
31 & 4.7602 & 65 & 4.8042 & 99 & 4.8644 \\
32 & 4.7607 & 66 & 4.8052 & 100 & 4.8665 \\
33 & 4.7636 & 67 & 4.8073 & & \\
34 & 4.7648 & 68 & 4.8089 & & \\
\hline & & & & & \\
\hline
\end{tabular}

Moreover, two levels with energies at 4.7830 and $4.7837 \mathrm{eV}$ appear in set I, whereas levels with energies at 4.7814 and $4.7836 \mathrm{eV}$ appear in set II. There are additional differences at higher vibrational energies as indicated by the thicker bars shown in Figure 1. By comparing our previours results for $\mathrm{H}_{3}$ [49] with the present results, we also observed that the GP effect has a more remarkable influence for $\mathrm{H}_{3}$ than for its isotopic variants. This is due to the fact that the crossing seam in $\mathrm{H}_{3}$ is characterized by $\theta_{s}=0$, whereas in $\mathrm{HD}_{2}$, the equation for the seam is defined by $\theta_{s}=0.5048 \mathrm{rad}, \phi_{s}=0$. The wider the region is on which the GP effect acts, the stronger the influence is on the vibrational spectra (see also Ref. [52]).

TABLE II Vibrational energy levels (in electronvolts) of $\mathrm{HD}_{2}$ calculated using the assumption $A(R)=\phi / 2$.

\begin{tabular}{|c|c|c|c|c|c|}
\hline 1 & 3.5939 & 35 & 4.7661 & 69 & 4.8124 \\
\hline 2 & 3.7591 & 36 & 4.7686 & 70 & 4.8136 \\
\hline 3 & 3.9150 & 37 & 4.7697 & 71 & 4.8153 \\
\hline 4 & 4.0614 & 38 & 4.7727 & 72 & 4.8180 \\
\hline 5 & 4.0851 & 39 & 4.7729 & 73 & 4.8194 \\
\hline 6 & 4.1158 & 40 & 4.7750 & 74 & 4.8211 \\
\hline 7 & 4.1981 & 41 & 4.7758 & 75 & 4.8230 \\
\hline 8 & 4.2285 & 42 & 4.7760 & 76 & 4.8245 \\
\hline 9 & 4.2571 & 43 & 4.7776 & 77 & 4.8259 \\
\hline 10 & 4.3248 & 44 & 4.7803 & 78 & 4.8277 \\
\hline 11 & 4.3606 & 45 & 4.7807 & 79 & 4.8297 \\
\hline 12 & 4.3870 & 46 & 4.7812 & 80 & 4.8309 \\
\hline 13 & 4.4407 & 47 & 4.7814 & 81 & 4.8312 \\
\hline 14 & 4.4475 & 48 & 4.7836 & 82 & 4.8337 \\
\hline 15 & 4.4810 & 49 & 4.7843 & 83 & 4.8356 \\
\hline 16 & 4.4974 & 50 & 4.7865 & 84 & 4.8367 \\
\hline 17 & 4.5449 & 51 & 4.7872 & 85 & 4.8375 \\
\hline 18 & 4.5564 & 52 & 4.7878 & 86 & 4.8409 \\
\hline 19 & 4.5718 & 53 & 4.7889 & 87 & 4.8424 \\
\hline 20 & 4.5897 & 54 & 4.7907 & 88 & 4.8437 \\
\hline 21 & 4.6114 & 55 & 4.7917 & 89 & 4.8460 \\
\hline 22 & 4.6157 & 56 & 4.7922 & 90 & 4.8475 \\
\hline 23 & 4.6360 & 57 & 4.7944 & 91 & 4.8496 \\
\hline 24 & 4.6656 & 58 & 4.7964 & 92 & 4.8515 \\
\hline 25 & 4.6815 & 59 & 4.7971 & 93 & 4.8532 \\
\hline 26 & 4.6852 & 60 & 4.7988 & 94 & 4.8563 \\
\hline 27 & 4.7037 & 61 & 4.8007 & 95 & 4.8578 \\
\hline 28 & 4.7115 & 62 & 4.8021 & 96 & 4.8562 \\
\hline 29 & 4.7193 & 63 & 4.8025 & 97 & 4.8577 \\
\hline 30 & 4.7602 & 64 & 4.8046 & 98 & 4.8587 \\
\hline 31 & 4.7607 & 65 & 4.8063 & 99 & 4.8611 \\
\hline 32 & 4.7636 & 66 & 4.8077 & 100 & 4.8639 \\
\hline 33 & 4.7648 & 67 & 4.8090 & & \\
\hline 34 & 4.7650 & 68 & 4.8104 & & \\
\hline
\end{tabular}




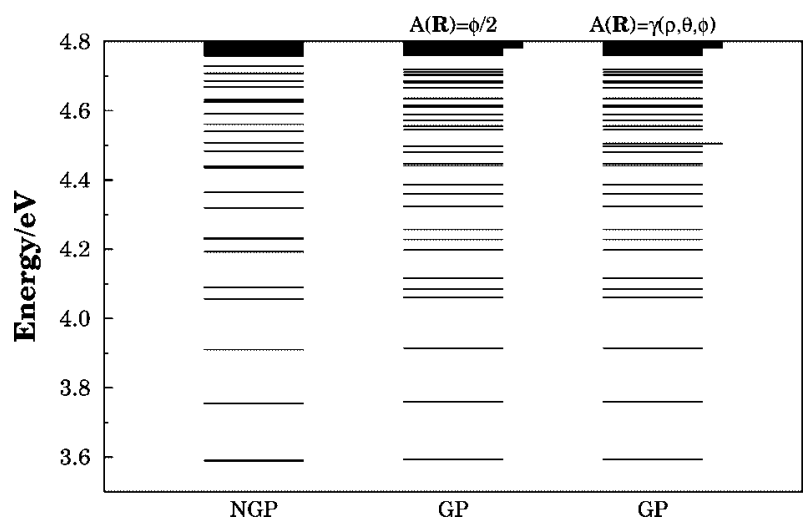

FIGURE 1. Comparison of the lowest "cone" states of $\mathrm{HD}_{2}$ calculated without consideration (NGP) and with consideration (GP) of the geometric phase effect. Shown by the thicker lines indicated the extra levels that arise in the two sets of GP calculations. See the text.

\section{Conclusions}

In the present work, we used a recently proposed GBO equation to study the GP effect in the vibrational spectra of $\mathrm{HD}_{2}$. Numerical calculations have demonstrated that there are significant differences between the results calculated from this GBO formulation and those based on the assumption that $A(\mathbf{R})=\phi / 2$. We also stress that using the formalism of the present work allows us to avoid the difficulties usually encountered when investigating the GP effect in nonsymmetric isotopomers of $X_{3}$. Indeed, we no longer need to determine the boundary line $\theta_{s}$, which defines the position of the crossing seam (and hence the closed loops for which the nuclear wave functions should change sign) in massdependent hyperspherical coordinates [25, 52].

\section{ACKNOWLEDGMENTS}

This work was supported by the Fundação para a Ciência e Tecnologia, Portugal, under program PRAXIS XXI.

\section{References}

1. Herzberg, G.; Longuet-Higgins, H. C. Faraday Discuss Chem Soc 1963, 35, 77.

2. Born, M.; Oppenheimer, J. R. Ann Phys 1927, 84, 457.

3. Jahn, H. A.; Teller, E. Proc R Soc London Ser A 1937, 161, 220.

4. Longuet-Higgins, H. C.; Öpik, U.; Pryce, M. H. L.; Sack, R. A. Proc R Soc London Ser A 1958, 244, 1.

5. Longuet-Higgins, H. C. Adv Spectrosc 1961, 2, 429.
6. Child, M. S.; Longuet-Higgins, H. C. Phil Trans R Soc London Ser A 1961, 254, 259.

7. Longuet-Higgins, H. C. Proc R Soc London Ser A 1975, 344, 147.

8. Köppel, H.; Domcke, W.; Cederbaum, L. S. Adv Chem Phys 1984, 72, 59.

9. Mead, C. A.; Truhlar, D. G. J Chem Phys 1979, 70, 2284.

10. Mead, C. A. Chem Phys 1980, 49, 23.

11. Mead, C. A. J Chem Phys 1980, 72, 3839.

12. Berry, M. V. Proc R Soc London Ser A 1984, 392, 45.

13. Aharonov, Y.; Anandan, J. Phys Rev Lett 1987, 58, 1593.

14. Aharonov, Y.; Bohm, B. Phys Rev 1959, 115, 485.

15. Mead, C. A. Rev Mod Phys 1992, 64, 51.

16. Yarkony, D. R. Rev Mod Phys 1996, 68, 985.

17. Varandas, A. J. C.; Yu, H. G. J Chem Soc Faraday Trans 1997, 93, 819 .

18. Kendrick, B. K. Phys Rev Lett 1997, 79, 2431.

19. Busch, H.; Dev, V.; Eckel, H.-A.; Kasahara, S.; Wang, J.; Demtröder, W.; Sebald, P.; Meyer, W. Phys Rev Lett 1998, 81, 4584.

20. Varandas, A. J. C.; Yu, H. G.; Xu, Z. R. Mol Phys 1999, 96, 1193.

21. Varandas, A. J. C.; Xu, Z. R. Int J Quantum Chem 1999, 75, 89.

22. Lepetit, B.; Peng, Z.; Kuppermann, A. Chem Phys Lett 1990, 166, 572.

23. Lepetit, B.; Kuppermann, A. Chem Phys Lett 1990, 166, 581.

24. Wu, Y.-S. M.; Lepetit, B.; Kuppermann, A. Chem Phys Lett 1991, 186, 319.

25. Kuppermann, A.; Wu, Y.-S. M. Chem Phys Lett 1993, 205, 577.

26. Kuppermann, A.; Wu, Y.-S. M. Chem Phys Lett 1995, 241, 229.

27. Kendrick, B. K.; T Pack, R. J Chem Phys 1996, 104, 7475.

28. Kendrick, B. K.; T Pack, R. J Chem Phys 1996, 104, 7502.

29. Kendrick, B. K.; T Pack, R. J Chem Phys 1997, 106, 3519.

30. Baer, M. J Chem Phys 1998, 109, 891.

31. Varandas, A. J. C.; Tennyson, J.; Murrell, J. N. Chem Phys Lett 1979, 61, 431.

32. Wu, Y.-S. M.; Kuppermann, A. Chem Phys Lett 1993, 201, 178.

33. Kuppermann, A.; Wu, Y.-S. M. Chem Phys Lett 1993, 213, 636.

34. Billing, G. D.; Marković, N. J Chem Phys 1993, 99, 2674.

35. Varandas, A. J. C.; Xu, Z. R. In Topics of Current Computational Chemistry; Bader, R.; Montero, L., Eds.; to be published.

36. Varandas, A. J. C. Adv Chem Phys 1988, 74, 255.

37. Varandas, A. J. C. In Reaction and Molecular Dynamics; Laganá, A.; Riganelli, A., Eds.; Lecture Notes in Chemistry; Springer-Verlag: Berlin, 2000; vol. 75, p. 33.

38. Varandas, A. J. C.; Pais, A. A. C. C. J Chem Soc Faraday Trans 1993, 89, 1511.

39. Pais, A. A. C. C.; Nalewajski, R. F.; Varandas, A. J. C. J Chem Soc Faraday Trans 1994, 90, 1381. 
40. Yu, H. G.; Smith, S. C. Ber Bunsen-Ges Phys Chem 1997, 101, 400.

41. Cuccaro, S. A.; Hipes, P. G.; Kuppermann, A. Chem Phys Lett 1989, 157, 440.

42. Sadeghi, R.; Skodje, R. T. J Chem Phys 1993, 99, 5126.

43. Skodje, R. T.; Sadeghi, R.; Köppel, H.; Krause, J. L. J Chem Phys 1994, 101, 1725.

44. Wu, Y.-S. M.; Kuppermann, A. Chem Phys Lett 1995, 235, 105.

45. Sadeghi, R.; Skodje, R. T. J Chem Phys 1995, 102, 193.

46. Sadeghi, R.; Skodje, R. T. J Phys Rev A 1995, 52, 1996.

47. Varandas, A. J. C.; Yu, H. G. Chem Phys Lett 1996, 259, 336.

48. Varandas, A. J. C.; Xu, Z. R. Chem Phys Lett 2000, 316, 248.

49. Varandas, A. J. C.; Xu, Z. R. J Chem Phys 2000, 112, 2121.

50. Xu, Z. R.; Baer, M.; Varandas, A. J. C. J Chem Phys 2000, 112, 2746.

51. Kuppermann, A. Chem Phys Lett 1975, 32, 374.

52. Xu, Z. R.; Varandas, A. J. C. Int J Quantum Chem 2000, 80, 454.

53. Baer, M.; Englman, R. Chem Phys Lett 1997, 265, 105.

54. Baer, M. J Chem Phys 1997, 107, 2694.

55. Kendrick, B. K.; Mead, C. A.; Truhlar, D. G. J Chem Phys $1999,110,7594$.

56. Baer, M. Chem Phys Lett 1975, 35, 112.

57. Baer, M. Chem Phys 1976, 15, 49.

58. Baer, M. Mol Phys 1980, 40, 1011.

59. Baer, M. In Theory of Chemical Reaction Dynamics; Baer, M., Ed.; CRC Press: Boca Raton, FL, 1985; Vol. II, pp. 243244.

60. Baer, M.; Englman, R. Mol Phys 1992, 75, 293.
61. Pacher, T.; Cederbaum, L. S.; Köppel, H. Adv Chem Phys 1993, 84, 293.

62. Domcke, W.; Stock, G. Adv Chem Phys 1997, 100, 1.

63. Yarkony, D. R. J Chem Phys 1996, 105, 10456.

64. Sadygov, R. G.; Yarkony, D. R. J Chem Phys 1998, 109, 20.

65. Matsunaga, N.; Yarkony, D. R. J Chem Phys 1997, 107, 7825.

66. Chaban, G.; Gordon, M. S.; Yarkony, D. R. J Phys Chem 1997, 101A, 7953.

67. Varandas, A. J. C. J Chem Phys 1997, 107, 867.

68. Varandas, A. J. C.; Voronin, A. I.; Caridade, P. J. S. B. J Chem Phys 1998, 108, 7623.

69. Preston, R. K.; Tully, J. C. J Chem Phys 1971, 54, 4297.

70. Grimbert, D.; Lassier-Govers, B.; Sidis, V. Chem Phys 1988, $124,187$.

71. Gianturco, F.; Palma, A.; Schnider, F. Chem Phys 1989, 137, 177.

72. Varandas, A. J. C.; Xu, Z. R. Chem Phys 2000, 259, 173.

73. Hobey, W. D.; McLachlan, A. D. J Chem Phys 1960, 33, 1695.

74. Smith, F. T. Phys Rev 1969, 179, 113.

75. Baer, R.; Charutz, D. M.; Kosloff, R.; Baer, M. J Chem Phys 1996, 105, 9141.

76. Baer, M. J Chem Phys 1997, 107, 10662.

77. Sjöqvist, E.; Hedström, M. Phys Rev A 1997, 56, 3417.

78. Polavieja, G. G.; Sjöqvist, E. Am J Phys 1998, 66, 431.

79. Johnson, B. R. J Comput Phys 1973, 13, 445.

80. Carter, S.; Meyer, W. J Chem Phys 1990, 93, 8902.

81. Carter, S.; Meyer, W. J Chem Phys 1994, 100, 2104.

82. Whitten, R. C.; Smith, F. T. J Math Phys 1968, 9, 1103.

83. Wei, H. J Chem Phys 1997, 106, 6885. 